\title{
CURRENT COMPUTATIONAL TRENDS IN EQUIPMENT PROGNOSTICS
}

\author{
J. Wesley HINES, Alexander USYNIN \\ Nuclear Engineering Department, University of Tennessee, \\ Knoxville, TN 38996, USA \\ E-mail:jhines2@utk.edu \\ http://www.utk. edu
}

Received October $12^{\text {th }}, 2007$

Revised October $17^{\text {th }}, 2007$

\begin{abstract}
The article overviews current trends in research studies related to reliability prediction and prognostics. The trends are organized into three major types of prognostic models: failure data models, stressor models, and degradation models. Methods in each of these categories are presented and examples are given. Additionally, three particular computational prognostic approaches are considered; these are Markov chainbased models, general path models, and shock models. A Bayesian technique is then presented which integrates the prognostic types by incorporate prior reliability knowledge into the prognostic models. Finally, the article also discusses the usage of diagnostic/prognostic predictions for optimal control.
\end{abstract}

Keywords: prognostics, reliability, Markov chain models, general path model, shock model, optimal control.

\section{Introduction}

Prognostics is an important aspect of an equipment surveillance system. Such a system is usually made up of several modules which use equipment related data to perform monitoring, diagnostics, and prognostics (see Fig. 1). While the monitoring and diagnostics (identification) portions have been well established for several decades, the prognosticsrelated techniques have recently attracted much attention in many research studies. The reason of the growing interest in the development of prognostic methods is that the prognostic requirements for modern engineering systems and mission- and safety-critical components have become quite ambitious and present many challenges to the system design teams. Many papers describe the monitoring, detection and identification functions of surveillance; two recent articles focusing on empirical models and module integration are by Garvey ${ }^{1}$ and Hines $^{2}$. Prognostic modules are usually developed to predict one of several related measures:

1. Remaining Useful Life (RUL): the amount of time, in terms of operating hours, cycles, or other measures the component will continue to meets its design specification.

2. Time to Failure (TTF): the time a component is expected to fail (no longer meet its design requirements).

3. Probability of Failure (POF): the failure probability distribution of the component.

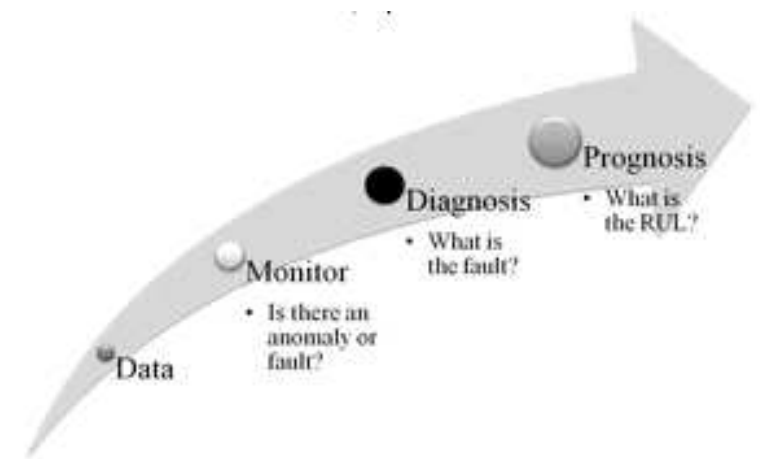

Fig. 1. Typical Equipment Surveillance System

These terms are very similar and the authors do not attempt to differentiate between the first two. Since the time the failure event occurs cannot be 
precisely predicted, a failure probability distribution results. Ideally, this distribution is constructed; however, point-wise failure-time estimates are more common. When point-wise estimates are given, a measure of uncertainty or a confidence interval around the prediction is desirable.

\subsection{Motivation}

Prognostics has emerged as an alternative to traditional reliability prediction, run-to-failure, and scheduled maintenance. Traditional approaches to system and component reliability are to be questioned since in many engineering applications the intrinsic lifespan of components and interconnections becomes significantly shorter than that of the systems within which they are used ${ }^{3}$. For example, the assumptions of essentially unlimited life and constant failure rate for electronics should be reviewed. System designers have traditionally assumed that the increasing failure rate portion of the well-known "bathtub" reliability curve is unreachable and not a concern in life cycle operations. This assumption has been historically correct, since component lifetime has traditionally been longer than the system's expected life. However, the advent of electronic components whose life is not longer than the system life makes the constant failure rate assumption invalid ${ }^{4}$.

In maintaining a fleet of complex engineering systems one can identify many needs such as maximum assets availability, very low rate of Returned Tested OK components, minimal periodic inspections, low number of spare items, accurate parts lifespan tracking, minimum false alarms, etc. ${ }^{5}$ Maintainers need to have the ability to accurately predict future equipment health status and to anticipate problems and maintenance routines before downtime occurs. Predictive capabilities would enable the maintainer to execute a very beneficial maintenance strategy based on future expected equipment condition. Some of the benefits provided by such an "on-condition" based maintenance are

- less time spent on inspection,

- optimized maintenance planning,

- improved fault detection, and

- increased asset availability.

Prognostic capabilities using existing monitoring systems, data, and information will enable more accurate equipment risk assessment and provide a basis for answering operational questions such as:
- Should we continue to operate or immediately shutdown for maintenance?

- Can we change operations (speed, load, stress) to continue operations to the next maintenance opportunity?

- Will the equipment have high probability of safe operation for the planned mission?

Prognostics founded in root cause analysis allows accurate physics-based diagnostic and prognostic determinations for nuclear plant equipment to be derived. Some research studies for understanding and controlling the aging processes of safetycritical nuclear plant components are currently in progress 6,7 .

\section{Prognostics Overview}

Prognostics methods can be categorized by their architecture, how they operate, the results they produce, or through several other means. An approach that may be most instructive is to categorize them by the type of information they use. Three prognostic method types are defined this way:

\section{Type I: Time-to-Failure Data-Based}

These methods consider historical time to failure data which are used to model the failure distribution. They estimate the life of an average component under average usage conditions. The most common method is Weibull Analysis ${ }^{8}$.

Type II: Stress-Based

These methods also consider environmental stresses (e.g. temperature, load, vibration, etc.) under which the component operates. They estimate the life for an average component under the given usage conditions. A common method is the Proportional Hazards Model ${ }^{9}$.

\section{Type III: Effects-Based}

These methods also consider the measurable or inferred component degradation. An example is the General Path Model ${ }^{10}$.

Figure 2 provides a graphical representation of the three prognostic methods. The most common type is Type I which is the initial topic of most reliability engineering texts such as those by Ebeling ${ }^{11}$, Elsayed ${ }^{12}$, Barlow and Proschan ${ }^{13}$ or Meeker and Escobar ${ }^{14}$. These books begin with the development of failure time methods and then progress to more advanced methods that use sensed information. The following sections will provide more details on each of these method types. 


\subsection{Type I: Failure Data-Based Prognostics}

In applications where failure rates of non-critical items are relatively low and the usage of advanced prediction models is constrained due to sensory equipment limitations, failure data-based prognostics is the only candidate to predict reliability. This group of methods estimates failure data density functions with various parametric or non-parametric models.

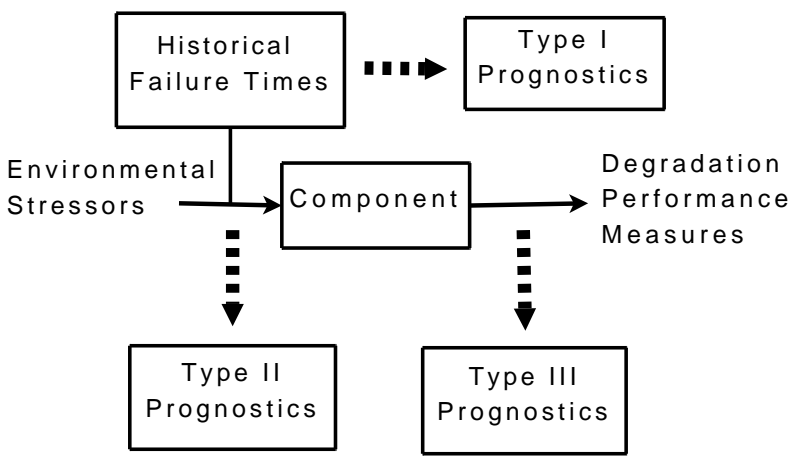

Fig. 2. Prognostic Method Types

Failure data analysis is based on gathering information about how long the item operates before failure. Statistics collected from a large sample of similar items are estimated to draw conclusions regarding time-to-failure for a typical item. In reliability analysis, the item's lifetime is modeled considering only a static probability distribution that does not take into account condition data observed at the particular object of interest. The lifetime probability distribution is given by

$$
F(t \mid \Theta)=P(T \leqslant t)
$$

where $t$ is some time, $T$ is a random variable representing failure time, and $\Theta$ is a vector of parameters.

The simplest parametric model is the exponential model which is applicable to constant failure rate components:

$$
F(t \mid \lambda)=1-\exp (-\lambda t)
$$

These components have an equal probability of failing for each instant of time. They do not degrade or wear out and are characterized by a single parameter: the constant failure rate $\lambda(t)=\lambda$. Hazard rate is commonly used in place of failure rate Probably the most common parametric model is the Weibull distribution. This model is used because it is flexible enough to model a variety of failure rates. The formula for the failure rate (eqn. (1)) is a two parameter model with a shape parameter $(\beta)$ and a characteristic life $(\theta)$.

$$
\lambda(t)=\frac{\beta}{\theta}\left(\frac{t}{\theta}\right)^{\beta-1}
$$

These two parameters provide the modeling flexibility for components exhibiting an increasing failure rate $(\beta>1)$, a constant failure rate $(\beta=1)$, and a decreasing failure rate $(\beta<1)$. With the correct choice of shape parameter, the Weibull distribution does a good job of modeling the exponential, normal, or Rayleigh distributions. Example of different shape parameters are given in Fig. 3.

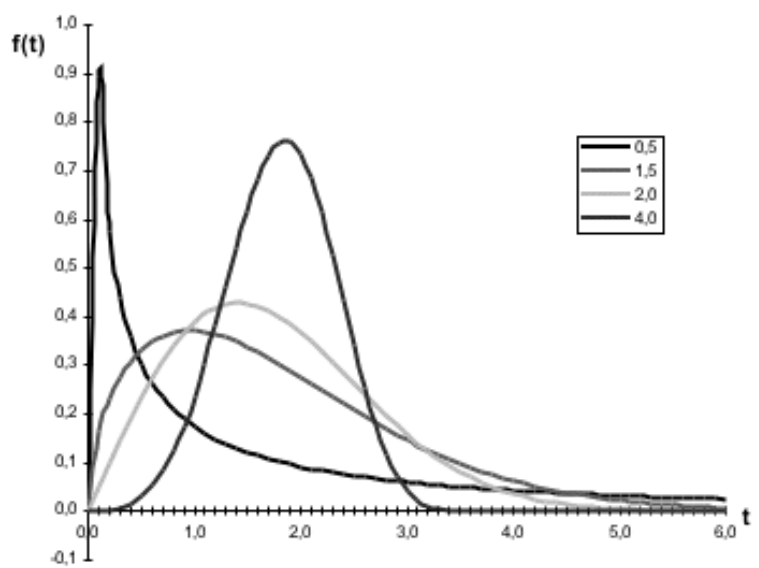

Fig. 3. Weibull failure distributions with different shape parameters

Additional information on Weibull modeling can be found in a multitude of texts one of which is the New Weibull Handbook ${ }^{8}$.

\subsection{Type II: Stress-Based Prognostics}

A readily apparent disadvantage of reliability databased prognostics is that it does not consider the operating condition under which a specific component is used. It provides a failure distribution for the average component operating under average conditions. However, components operating under harsh conditions would be expected to fail sooner and components operating under mild conditions to last longer. A group of prognostic methods that take the operating conditions under consideration are aptly named stress-based prognostics.

The simplest class of methods for stress-based prognostics is failure-time linear regression models. These models use prior observations of explanatory variables such as stress, temperature, or voltage and the response variable, which is usually the failure time, to predict the life of a component. The 
proportional hazards model (PHM), developed by Cox ${ }^{15}$, is a technique that merges failure time data and stress data. The model uses environmental condition information, termed covariates $\left(z_{j}\right)$, to modify a baseline hazard rate $\left(\lambda_{0}(t)\right)$ to form a new hazard rate:

$$
\lambda(t ; z)=\lambda_{0}(t) \exp \left(\sum_{j=1}^{q} \beta_{j} z_{j}\right)
$$

Failure data collected at covariate operating conditions are used to solve for the parameters $\left(\beta_{j}\right)$ using Maximum Likelihood Estimation (MLE) algorithm. A basic assumption of the PHM is that the covariates are multiplicative. The multiplicative effect of the covariates on the baseline is such that when the ratio of two cases is evaluated at some time, their hazard rates are proportional. The baseline hazard is the hazard rate when covariates have little or no influence on the failure rate.

\subsection{Type III: Effects-Based Prognostics}

Effects-based prognostics uses degradation measures to form a prognostic prediction. A degradation measure is a scalar or vector quantity that numerically reflects the current ability of the system to perform its designated functions properly. It is a quantity that is correlated with the probability of failure at a given moment. A degradation path is a trajectory along which the degradation measure is evolving in time towards the critical level corresponding to a failure event.

The degradation measure does not have to be a directly measured parameter, it could be a function of several measured variables that provide a quantitative measure of degradation. It could also be an empirical model prediction of the degradation that cannot be measured. For example, pipe wall thickness may be an appropriate degradation parameter but there may not be an unobtrusive method to directly measure it. However, there may be related measurable variables that can be used to predict the wall thickness. In this case the degradation parameter is not a directly measurable parameter but a function of several measurable parameters.

Many effects-based prognostics models track the degradation (damage) as a function of time and predict when the total damage will exceed a predefined threshold that defines failure. Cumulative damage is defined to be irreversible accumulation of damage in components under cyclical loadings. There are several mathematical approaches to model cumulative damage.

- Markov Chain-based Models
- Shock Models

- General Path Models

Markov Chain Prognostic Models are discrete in the time domain and in the degradation measure domain. For each duty cycle, there is a non-zero probability of receiving a unit-size damage. The model is usually formulated as a probabilistic simulation of past and future degradation ${ }^{16}$. If the degradation is directly measurable, then the simulation is only performed for the future. The model has several parameters which can be estimated from historical degradation and failure data:

- Probability of a damage occurrence in a duty cycle

- The magnitude of the damage (usually a unit-size damage is assumed)

- The critical damage level (Failure Threshold)

Figure 4 shows an example Markov Chain prognostic model. The area marked by the diagonal upward hatching is a collection of degradation pathways that grow towards the failure threshold over time. If the actual degradation is measurable, then the model can be used to simulate future pathways from the current state.

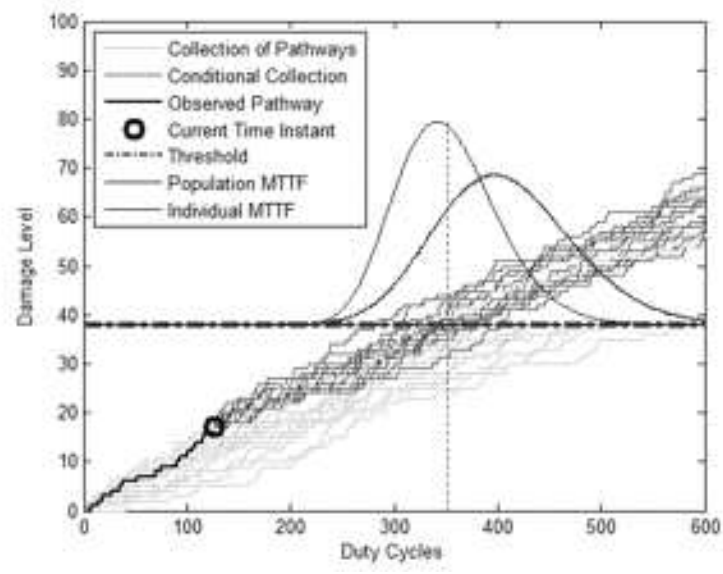

Fig. 4. Markov Chain Prognostic Model Example

These are represented by the dot-filled area. The collection of degradation paths can be used to predict the failure distribution (POF). In the figure, Distribution $F_{1}$ represents the population failure distribution while Distribution $F_{2}$ is the predicted distribution for the individual. One can see that the individual failure density has a lower variance (less uncertainty) than the population-based distribution. This represents the advantage of using individualbased prognostics: reduction in the uncertainty of the predicted RUL. As the degradation approaches 
the failure threshold, the RUL prediction becomes more exact.

Shock Models are used predict the RUL for systems which are subject to randomly arriving shocks, which deliver some damage of a random magnitude ${ }^{17}$. They are continuous in the time and the degradation measure domains. Shock models have several important parameters that are estimated from historical failure data:

- Random Time between successive shocks,

- Random Magnitude of Shocks

- The critical failure threshold

Figure 5 shows an example shock model. The method is similar to the Markov Chain model but the time between shocks and the shock magnitudes are continuous random variables.

General Path Models (GPM) were first proposed by $\mathrm{Lu}$ and Meeker ${ }^{10}$ and use measurable degradation data and predict future degradation over time. The GPM assumes that the degradation is a function of time, duty cycles, or some other measure. Extrapolation of this degradation function was used for RUL estimation by Upadhyaya, et al. ${ }^{18}$ Depending on the model functional relationship, the model could have several parameters. A linear model has two parameters:

- Critical Threshold

- Random Degradation Rate

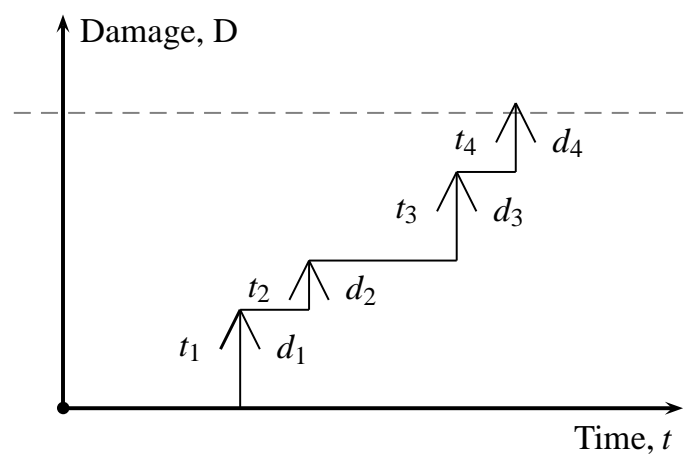

Fig. 5. Shock Model Example

The linear model is represented by Eq. (3) in which the damage is a function of time.

$$
\mathrm{D}(t)=\alpha t+\varepsilon
$$

where $\varepsilon \simeq \mathrm{N}\left(0, \sigma_{\text {noise }}^{2}\right)$ is the error term.

Figure 6 shows a linear path model example. The model parameters are estimated using historical data and the RUL is calculated by measuring the current degradation and estimating the RUL using the linear model. Confidence intervals can be calculated using standard linear regression equations. The details of using linear path models for RUL prediction is given by Usynin ${ }^{19}$.

Other functional relationships may also be used.

Figure 7 shows a general path model in which the degradation path is not linear. In these cases in which degradation may increase with time or load cycles, a transformation may be derived to make the relationship linear, or the actual functional form can be predicted from the historical degradation paths. The observed degradation path, y, is given by Eq. (4)

$$
y_{i}=\eta\left(t, \varphi, \Theta_{i}\right)+\varepsilon
$$

where $\varphi$ is the vector of fixed effects (population) parameters and $\Theta_{i}$ is the vector of random (individual) effects for unit $i$. The Time-to-Failure (TTF) distribution is given by Eq. (5)

$$
\operatorname{Pr}\{T \leqslant t\}=F_{T}\left(t, \varphi, G_{\Theta}(\cdot), \mathrm{D}, \eta\right)
$$

where $G_{\Theta}$ is the distribution of $\Theta_{i}$ and D is the critical threshold.

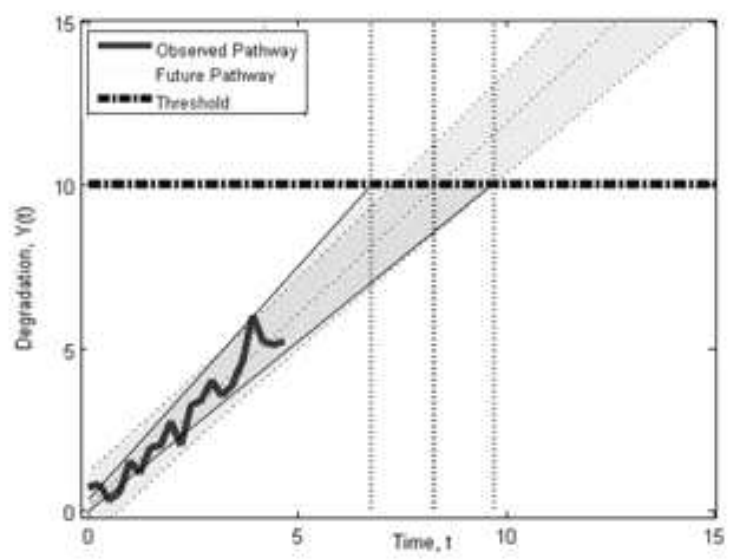

Fig. 6. Linear Path Model Example

A common method for integrating prior population based historical data with current individual data is Bayesian updating. A complete discussion of Bayesian statistics is available in Carlin and Louis ${ }^{20}$. The following notation is used to describe the Bayesian procedure.
$\Theta$
- model parameter to estimate
DATA - available observations
L(DATA $\mid \Theta)$ - likelihood of DATA
$f(\Theta) \quad$ - the prior density of $\Theta$
$f(\Theta \mid$ DATA $)$ - the posterior PDF of $\Theta$ 
Figure 8 shows the basic Bayesian updating procedure. First, historical data is used to estimate the model parameter. Next, new data is collected and used to update the model resulting in a new posterior distribution of the parameters. This posterior is used as the new prior distribution. Lastly, when new data is collected, it is used update the parameter distribution again.

As an example, a number of degradation paths may have been collected that are modeled with a linear model. The one unknown parameter is the slope parameter, and the historical linear fits can be used to create a probability distribution for the slope parameter. Without any collected data, one would expect the new individual to have the same traits as the historical population; thus, this historical behavior provides a prior distribution. As new data is collected, the prediction of the distribution is individualized so that it is tailored to the current individual component. Over time, the measured data overwhelms the prior distribution and the predicted slope is that of the data.

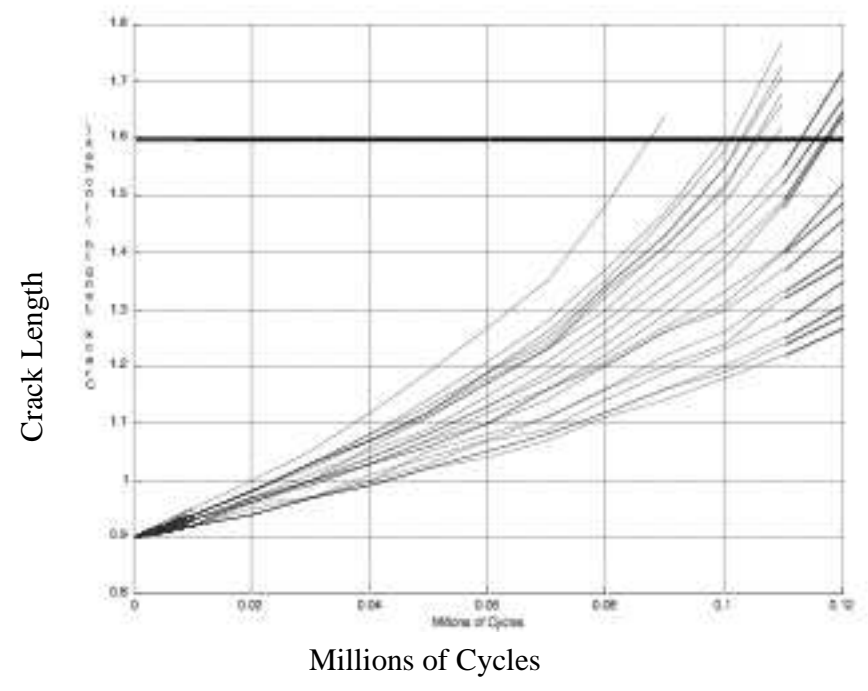

Fig. 7. General Path Model Example

\subsection{Combined Prognostics Model Types}

Some prognostic model architectures can make use of a combination of historical failure data, environmental data, and effects based data. An example of a combined prognostics architecture is the life consumption monitoring (LCM) methodology introduced by Ramakrishnand and Pecht ${ }^{21}$.

A brief review of the LCM procedure is given in Mishra ${ }^{22}$. The LCM is defined to be a prognostic methodology that consists of the following steps:

1) Failure modes, mechanisms and effects analysis,

2) Virtual reliability assessment,

3) Monitoring critical parameters,

4) Raw data simplification,

5) Stress and damage analysis,

6) RUL prognosis.

Each step is briefly described and references to the mathematical models involved are given. Two case studies were performed to demonstrate the proposed methodology. The objects of interest in the both studies were two identical printed circuit boards (PCB) placed under the hood of a car. The PCB's were subject to various stress conditions. Temperature and vibration were identified to be the strongest affecting factors. A failure modes and mechanisms analysis revealed seven different failure modes such as electrical short between traces, short between windings in the inductors populating the PCB's, change in electrical resistance due to solder joints degradation.

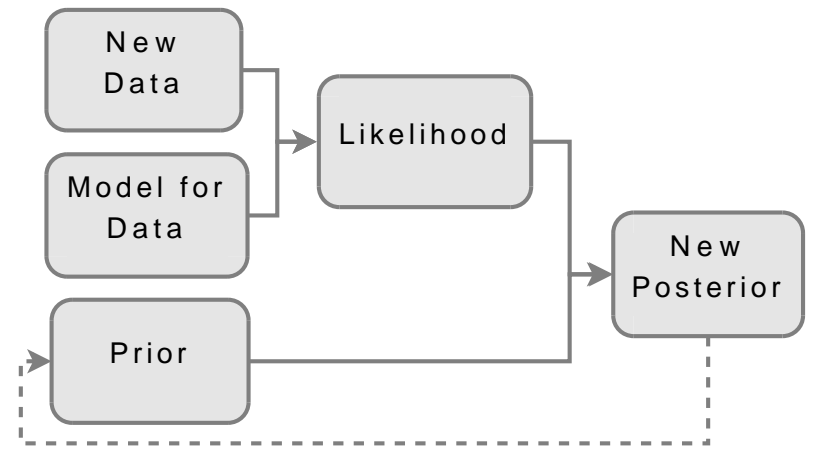

Fig. 8. Bayesian Updating Methodology

Virtual reliability assessment revealed that the failure mode having the shortest time-to-failure was a solder joint fatigue. The conducted assessment predicted 34 days to failure based on solder joint fatigue.

In recently published prognostics research, a great deal of attention has been focused on the use of machine learning techniques such as artificial neural networks, fuzzy logic-based models, classification and pattern recognition methods $23,24,25,26$.

A variety of neural network modifications have been applied to construct a prognostic framework.

Wang and Vachtsevanos ${ }^{27}$ use dynamic wavelet neural networks as the prognostic system reasoner. A combination of radial basis function neural networks and rule extractors is applied to gas turbine engine prognostics by Brotheron ${ }^{28}$. A Bayesian be- 
lief network is a main tool in Health Management System for avionics proposed by Parker ${ }^{29}$.

\subsection{Gaps in Prognostics-related Research and Possible Future Directions}

In recently published prognostics-related research much attention has been paid to the various models and approaches ranging from ordinary least squares regression to wavelet-based neural networks that aim primarily to producing an accurate prediction of future reliability conditions of the object of interest. To the authors' knowledge, only few studies published in the open literature have considered the use of prognostic information for constructing an optimal preventive maintenance strategy, these are by Gertsbakh ${ }^{30}$ and Jardine and Tsang ${ }^{31}$. The studies agree in that preventive maintenance based on the usage of diagnostic information periodically or continuously collected on the system of interest is expected to be more efficient than a preventive maintenance strategy based on the time-to-failure distribution. However, the higher efficiency comes at the expense of having a prognostic model characterizing statistical properties of the random process $\eta(t)$, which reflects damage accumulation (degradation) in the system.

Considering capabilities to continuously assess and predict reliability aspects of the system, the practitioner may be interested in how the prognostic information can improve the system control in terms of availability and cost reduction.

The research field related to the use of prognostic models for optimal control remains wide-open. Some researchers and practitioners have outlined the main directions to follow and issues to address in regards to the prognostics-based optimal control ${ }^{32,33}$. However, there is a significant lack of specific control methods and approaches which would be suitable for dealing with uncertain conditions imposed by highly random environmental conditions, variability in operational loadings and imperfect reliability models.

In the presence of a large variety of diagnostic information available online, it would be highly desirable to develop methods and approaches for incorporating the prognostic information into the optimal control of the system. The following qualitative example illustrates the idea of prognostic-based control.

Consider a system assigned to complete some mission subject to some time constraints. The system performance is numerically quantified by the system performance rate, which can be thought of as the metric characterizing how fast the system is able to accomplish the mission. Quality-related aspects of accomplishing the mission are out of this simple example's scope.

The system is assumed to be subject to degradation. While in operation the system degrades at some degradation rate, which is a function of a) the system current performance rate, and b) the current environmental conditions.

The performance rate, at which the system is operating in particular environmental conditions imposes some degradation rate that shapes the system degradation profile. Apparently severe environmental conditions and a high-performance rate will cause the system to degrade fast. On the contrary, normal environmental conditions and moderate performance rates cause the system to degrade relatively slowly.

The environmental conditions evolve independently from the system and are considered to be totally random and uncontrollable. The performance rate is assumed to be the only means to control the system performance.

In this setting, the practitioner wants to accomplish the mission at the lowest expense in terms of degradation acquired by the system. Running the system at a high performance rate minimizes the time needed to finish the mission; thus, meeting the time constraints. However, the high performance rate imposes a high degradation rate, especially in the case of severe environmental conditions. This can cause the system to fail due to wear-out before the mission is accomplished. On the other hand, a low or moderate performance rate can hinder the mission progress, and eventually cause the system to fail in accomplishing the mission since the time constraints are not met. It can be concluded that one should find an optimal performance rate, following which the system will meet the reliability requirements and time-related constraints as well. An example of this case may be the drilling of an oil well when inbound weather conditions limit the time on task.

Since the degradation rate is subject to random fluctuations, there is no one single value of the optimal performance rate that would provide an acceptably good result for any sequence of encountered environmental conditions. The practitioner rather needs to have an optimal control policy that would select a control action (the performance rate) optimally chosen for each combination of the environmental conditions and the degradation level the system has attained. One obvious solution to the control 
policy problem would be that the practitioner would choose the performance rate such that the standardized mean performance rate would be larger than or equal to the standardized mean degradation rate. The standardized rate means the rate defined for a percent value ranging from 0 to 100 . Thus the standardized mean performance rate is the volume (percentage) of mission-related work, which in average is performed within a unit of time. The standardized mean degradation rate is the damage (degradation) acquired by the system in a time unit.

$$
\begin{aligned}
& \text { Mean Performance Rate }=\mathrm{E}_{t}[\mathrm{~W}(t+\Delta t)-\mathrm{W}(t) / \Delta t] \\
& \text { Mean Degradation Rate }=\mathrm{E}_{t}[\mathrm{D}(t+\Delta t)-\mathrm{D}(t) / \Delta t]
\end{aligned}
$$

where $\mathrm{W}(t)$ is the percentage of the mission completed, $0 \% \leqslant \mathrm{~W}(t) \leqslant 100 \%, \mathrm{D}(t)$ is the degradation acquired by the system up to time $t, 0 \% \leqslant \mathrm{D}(t) \leqslant$ $100 \%$.

Following this control policy the performance rate is selected such that the newly updated average performance rate is larger than, or at least equal to, the mean degradation rate imposed by the chosen control action (performance rate). The deficiency of this control policy is that the mean degradation rate is assessed with some uncertainty attributed to the imperfectness of the prediction methods and/or uncertainty due to sensory equipment limitations. The errors in assessing the mean degradation rate can cause the chosen performance rate to significantly deviate from the optimal rate.

A more sophisticated example may include the presence of uncertainty in the performance rate that is due to the impact of the system degradation. Running a degraded piece of equipment would cause deviations from the normal performance profile such that the identical control actions performed in the initial and near-to-finish stages initiate different performance rates. The spread of deviations in the system performance profile is expected to significantly affect the control policy in terms of its optimality.

Concluding this section, the following statement is made. The usage of prognostic information for optimal control can greatly improve the assets operational availability and cost reduction. However, the problem of incorporating the prognostic information into the control process seems to be difficult and requires approaches that would handle a number of uncertainty-related issues and surpass the best of heuristic control strategies. A good candidate to resolve the problem is reinforcement learning (RL), which is a general algorithmic approach to stochastic optimal control problems. RL-based algorithms can perform with or without models of the system. The algorithms can be used online and offline as well. The distinctive feature of RL-based methods is that they tend to focus computation on areas in the system state space, where the control actions are likely to be taken.

\section{Conclusion}

This article presented a brief overview of current trends in prognostics-related research. A classification of prognostic model types has been presented which is based on the types of data sources that are available in real-world applications. These data sources are a) historical records reflecting general reliability aspects of the entire population (or a large sample) of one-kind components, b) reliability data including external stresses affecting the system reliability, c) reliability data including degradation effects observed at the system of interest. It should be noted that there could be classifications based on some other criteria, for example, types of reliability models, (empirical versus physics-based models); however, one would probably choose a prognostic technique based on the data available and prognostic objectives.

The article has also outlined one possible direction in prognostics research regarding the use of predicted reliability information for optimal operational control. In the presence of a great deal of uncertainty attributed to random environment, variability in operational loads, and item-to-item variation of reliability properties, reinforcement learning-based algorithms seem to be an appropriate method for solving the optimal control problem for degrading equipment given imperfect prognostic information.

\section{References}

1. D.R. Garvey and J.W. Hines, An Integrated Fuzzy Inference Based Monitoring, Diagnostic, and Prognostic System, 61 ${ }^{\text {th }}$ Meeting of the Society for Machinery Failure Prevention Technology, Virginia Beach, Virginia, (April, 2007).

2. J.W. Hines, "Empirical Methods for Process and Equipment Condition Monitoring", 52 nd Annual Reliability and Maintainability Symposium (RAMS), Newport Beach CA, (Jan 23-26, 2006).

3. C. Wilkinson, D. Humphrey, B. Vermeire, and J. Houston, (2004), "Prognostic and health management for avionics", IEEE Proc. of Aerospace Conference, $\mathbf{5}$, 3435-3447 (March 2004. 
4. S. Huber, (2002), "Segmentation and Reliability", DMSMS 2002 Intel Conference, New Orleans LA, March 2002.

5. A. Hess, G. Calvello, and P. Frith, , "Challenges, Issues, and Lessons Learned Chasing the "Big P": Real Predictive Prognostics", Proc. of IEEE Aerospace Conference, 3610-3619 (March 5-12, 2005).

6. L.J. Bond, D.B. Jarrell, "Prognostics and CBM-A Scientific Crystal Ball", Proc. of ANS Meeting, 9-13 (January 2002).

7. L.J. Bond et al., (2000), "Online Intelligent SelfDiagnostic Monitoring For Next Generation Nuclear Plants", Technical Report for NERI, DE-FG0399SF9491, September 2000.

8. R.B. Abernethy, The New Weibull Handbook, $2^{\text {nd }}$ edn. Abernethy, North Palm Beach, 1996.

9. D.R. Cox, Regression Models and Life-Tables, J. of the Roy. Statis. Soc., Series B (Methodological), 34 (2) (1972) 187-220.

10. C.J. Lu and W.Q. Meeker, "Using Degradation Measures to Estimate a Time-to-Failure Distribution", Technometrics 35 (2), 161-173 (May 1993).

11. C.E. Ebeling, An Introduction to Reliability and Maintainability Engineering, Waveland Press, Inc., 1997, reissued 2005.

12. E.A. Elsayed, Reliability Engineering, Addision Wesley, 1996.

13. R.E. Barlow and F. Proschan, Mathematical Theory of Reliability, John Wiley and Sons, NY, 1965.

14. W.O. Meeker and L.A. Escobar, Statistical Methods for Reliability Data, John Wiley and Sons, 1998.

15. D.R. Cox and D. Oakes, Analysis of survival data, Chapman \& Hall, 1984.

16. J.L. Bogdanoff and F. Kozin, Probabilistic Models of Cumulative Damage, John Wiley and Sons, NY, 1985.

17. A. Gut, "Cumulative Shock Models", Adv. in Appl. Probab., 22 (2), 504-507, 1990.

18. B.R. Upadhyaya, M. Naghedolfeizi, and B. Raychaudhuri, "Residual Life Estimation of Plant Components", P/PM Technology, 22-29 (June 1994).

19. A. Usynin and J.W. Hines, Use of Linear Growth Models for Remaining Useful Life Prediction, MARCON 2007.

20. B.P. Carlin and T.A. Louis, Bayes and Empirical Bayes Methods for Data Analysis, $2^{\text {nd }}$ edn. Boca Raton, Chapman and Hall/CRC, 2000.
21. A. Ramakrishnand and M. Pecht, (2003), "A life consumption monitoring methodology for electronic systems", IEEE Trans. on Components and Packaging Technologies, 26 (3), 625-634, Sep. 2003.

22. S. Mishra, S. Ganesan, M. Pecht, and J. Xie, (2004), "Life Consumption Monitoring for Electronic Prognostics", Proc. of IEEE Aerospace Conference, 2004.

23. D. He, S. Wu, P. Banerjee, and E. Bechhofer, "Probabilistic Model Based Algorithms for Prognostics", Proc. of IEEE Aerospace Conf., 1-10 (2006).

24. W.G. Zanardelli, E.G. Strangas, H.K. Khalil, and J.M. Miller, "Wavelet-based Methods for the prognosis of mechanical and electrical failures in electric motors", Mechanical Systems and Signal Processing, 19, 411426 (2003).

25. S.W. Wegerich, A.D. Wilks, and R.M. Pipke, "Nonparametric Modeling of Vibration Signal Features for Equipment Health Monitoring", Proc. of IEEE Aerospace Conf., 7, 3113-3123 (2003).

26. T. Brotheron, et al., "A Testbed for Data Fusion for Engine Diagnostics and Prognostics", Proc. of IEEE Aerospace Conf., New York, 6, 3029-3042 (2002).

27. P. Wang and G. Vachtsevanos, "Fault Prognostics using Dynamic Wavelet Neural Networks", Artificial Intelligence for Engineering Design and Manufacturing, 15, 349-365 (2001).

28. T. Brotheron, G. Jahns, J. Jacobs, and D. Wroblewski, "Prognosis of Faults in Gas Turbine Engines", Proc. of IEEE Aerospace Conf., 6, 163-171 (2000).

29. B.E. Parker et al., "Helicopter Gearbox Diagnostics and Prognostics Using Vibration Signature Analysis", Proc. of International Society for Optical Engineering, 1965, 531-542 (1993).

30. I. Gertsbakh, Reliability Theory with Applications to Preventive Maintenance, Springer, Berlin, 2000.

31. A.K. Jardine and A.H. Tsang, Maintenance, Replacement, and Reliability: Theory and Applications, CRC Press, Boca Raton, 2006.

32. L. Tang, G.J. Kacprzynski, J.R. Bock, and M. Begin, "An Intelligent Agent-based Self-evolving Maintenance and Operations Reasoning System", Proc. of IEEE Aerospace Conf., Big Sky, MT, 2006.

33. G. Davis et al., Predictive and Prognosotic Controller for Wide Band Gap Power Conversion, Proc. of IEEE Aerospace Conf., Big Sky, MT, 2006. 